\title{
Virulence of an emerging respiratory pathogen, genus Pandoraea, in vivo and its interactions with lung epithelial cells
}

\begin{abstract}
Correspondence
Siobhán McClean

siobhan.mcclean@ittdublin.ie
\end{abstract}

Received 26 May 2010

Accepted 29 November 2010

\author{
Anne Costello, ${ }^{1,2}$ Gillian Herbert, ${ }^{1}$ Lydia Fabunmi, ${ }^{1}$ Kirsten Schaffer, ${ }^{3}$ \\ Kevin A. Kavanagh, ${ }^{4}$ Emma M. Caraher, ${ }^{1,2}$ Máire Callaghan ${ }^{1,2}$ \\ and Siobhán McClean ${ }^{1,2}$
}

\author{
${ }^{1}$ Centre of Microbial Host Interactions, ITT Dublin, Tallaght, Dublin 24, Ireland \\ ${ }^{2}$ Centre of Applied Science for Health, ITT Dublin, Tallaght, Dublin 24, Ireland \\ ${ }^{3}$ Department of Microbiology, St Vincent's University Hospital, Elm Park, Dublin, Ireland \\ ${ }^{4}$ Department of Biology, National University of Ireland, Maynooth, Co. Kildare, Ireland
}

\begin{abstract}
Pandoraea species have emerged as opportunistic pathogens among cystic fibrosis (CF) and non-CF patients. Pandoraea pulmonicola is the predominant Pandoraea species among Irish CF patients. The objective of this study was to investigate the pathogenicity and potential mechanisms of virulence of Irish P. pulmonicola isolates and strains from other Pandoraea species. Three patients from whom the $P$. pulmonicola isolates were isolated have since died. The in vivo virulence of these and other Pandoraea strains was examined by determining the ability to kill Galleria mellonella larvae. The P. pulmonicola strains generally were the most virulent of the species tested, with three showing a comparable or greater level of virulence in vivo relative to another CF pathogen, Burkholderia cenocepacia, whilst strains from two other species, Pandoraea apista and Pandoraea pnomenusa, were considerably less virulent. For all Pandoraea species, whole cells were required for larval killing, as cell-free supernatants had little effect on larval survival. Overall, invasive Pandoraea strains showed comparable invasion of two independent lung epithelial cell lines, irrespective of whether they had a CF phenotype. Pandoraea strains were also capable of translocation across polarized lung epithelial cell monolayers. Although protease secretion was a common characteristic across the genus, it is unlikely to be involved in pathogenesis. In conclusion, whilst multiple mechanisms of pathogenicity may exist across the genus Pandoraea, it appears that lung cell invasion and translocation contribute to the virulence of $P$. pulmonicola strains.
\end{abstract}

\section{INTRODUCTION}

Pandoraea species are characterized as non-spore-forming, catalase-positive, aerobic, Gram-negative rods with polar flagella, and are often mistakenly identified as Burkholderia cepacia complex (Bcc) or Ralstonia species (Schneider et al., 2006; Stryjewski et al., 2003). At the time of writing, the genus Pandoraea contains five described species, Pandoraea apista, Pandoraea pulmonicola, Pandoraea pnomenusa, Pandoraea sputorum and Pandoraea norimbergensis (Stryjewski et al., 2003; Vandamme et al., 2000), which have been isolated from patients with respiratory tract infections as well as from soil, water and food (Schneider et al., 2006). Its closest

Abbreviations: Bcc, Burkholderia cepacia complex; CF, cystic fibrosis; CFS, cell-free supernatant; CFTR, cystic fibrosis transmembrane regulator; FEV, forced expiratory volume; p.i., post-infection; TER, transepithelial resistance. phylogenetic relative is the genus Burkholderia and, like members of the genus Burkholderia, it has recently emerged as a pathogen in the cystic fibrosis (CF) population. CF is a genetically inherited disorder caused by mutations in the $\mathrm{CF}$ transmembrane regulator (CFTR), a chloride channel. The main source of morbidity and mortality for CF patients, however, is the bacterial pathogens that they encounter throughout their lives, including Pseudomonas aeruginosa and, to a lesser extent, Bcc. Although Pandoraea species have also been isolated from sputum samples of CF patients, there is still very little known about their mechanisms of pathogenicity or their roles in CF lung disease (LiPuma, 2003). In addition, Pandoraea isolates have been recovered from both CF and non-CF patients from a variety of clinical samples including blood, sputum, urine, the upper airways and lung tissue (Pimentel \& MacLeod, 2008). The recovery of Pandoraea isolates from the blood of patients indicates that 
this organism is capable of invading tissue (Atkinson et al., 2006; Stryjewski et al., 2003). Antibiotic therapy for infection is difficult due to the limited number of antibiotics to which these species are susceptible: tetracycline, imipenem and trimethoprim-sulfamethoxazole (Schneider et al., 2006).

A systematic review of 102 publications has recommended that CF patients colonized with either Pandoraea or Bcc species should be segregated from other non-colonized patients (Vonberg \& Gastmeier, 2005). However, the clinical significance of colonization with these organisms remains unclear (Atkinson et al., 2006) and there are limited and conflicting data available on the clinical outcome of patients colonized with Pandoraea. Two CF patients chronically colonized with $P$. apista were culturenegative following post-bilateral transplants after antibiotic therapy (Atkinson et al., 2006). However, apparent patientto-patient transmission of $P$. apista has been demonstrated, and four of the patients exhibited a decrease in lung function post-acquisition (Jørgensen et al., 2003). P. pnomenusa sepsis and subsequent death has been reported in a non-CF patient after a lung transplant (Stryjewski et al., 2003). A recent case report highlighted nine previous reports of Pandoraea bacteraemia, but only one of these was identified as being in a CF patient (Pimentel \& MacLeod, 2008).

The predominant Pandoraea species among Irish CF patients is $P$. pulmonicola. We have shown previously that the Irish $P$. pulmonicola isolates were unusual within the genus as they all showed an ability to invade human lung epithelial A549 cells in vitro (Caraher et al., 2008). The three patients from whom the $P$. pulmonicola isolates were obtained have since died. Therefore, the virulence of these $P$. pulmonicola isolates required further study. We compared a selection of these isolates with other strains in an in vivo virulence model, Galleria mellonella, and also examined the interactions between some of these $P$. pulmonicola isolates and lung epithelial cells in more detail. The G. mellonella model was chosen as it has been used previously to examine the virulence of a number of CF pathogens including Bcc, Pseudomonas aeruginosa and Aspergillus fumigatus (Kavanagh \& Reeves, 2004; Reeves et al., 2004; Seed \& Dennis, 2008). The non-Irish strains were all sourced from a commercial bacterial culture collection and were all originally isolates from CF lung (Table 1).

\section{METHODS}

Bacterial strains and cell lines. The origins of the Pandoraea strains examined in this study are listed in Table 1. The non-Irish isolates were purchased from the BCCM/LMG, University of Ghent, Belgium. Non-invasive Escherichia coli strain NCIB 9485 was used as a control in all experiments. All isolates were routinely grown on/in tryptic soy agar, tryptic soy broth, Luria-Bertani (LB) agar or LB broth at $37^{\circ} \mathrm{C}$. All cell lines were maintained in a humidified atmosphere at $37{ }^{\circ} \mathrm{C}$ in $5 \% \mathrm{CO}_{2}$. Calu-3 cells (sub-bronchial epithelial cells) were obtained from the European Collection of Cell Cultures and maintained as described previously (Shen et al., 1994).
The cell lines $16 \mathrm{HBE}^{-} \mathrm{o}^{-}$(human bronchial epithelial cells with functional CFTR) and CFBE410 ${ }^{-}$(CF bronchial epithelial cells) were kindly donated by Dr Dieter Gruenert (University of California, San Francisco, CA, USA) and maintained in fibronectin/vitrogen-coated flasks containing minimal essential medium supplemented with $5 \mathrm{mM}$ L-glutamine, $50 \mathrm{U}$ penicillin $\mathrm{ml}^{-1}, 50 \mu \mathrm{g}$ streptomycin $\mathrm{ml}^{-1}$ and $10 \%(\mathrm{v} / \mathrm{v})$ fetal bovine serum.

Determination of virulence in vivo using G. mellonella larvae. Sixth-instar larvae of G. mellonella were obtained from Livefoods Direct and stored in wood shavings in the dark at $15{ }^{\circ} \mathrm{C}$. Ninety larvae $(0.2-0.4 \mathrm{~g}$ each) were randomly chosen for each strain assayed, and all experiments were performed on three independent occasions. Bacterial cells were harvested by centrifugation at $4000 \mathrm{~g}$ for $10 \mathrm{~min}$ and washed in PBS. The $\mathrm{OD}_{600}$ was obtained and cultures were diluted to a standard of $0.1 \mathrm{OD}_{600}$. Bacterial cultures were then serially diluted to $10^{-7}$. The bioburden was ascertained postinjection by plate counts of dilutions from $10^{-4}$ to $10^{-7}$, plated in triplicate. Ten larvae were inoculated with $20 \mu \mathrm{l}$ each dilution through the last pro-leg into the haemocoel using a $0.3 \mathrm{ml}$ Myjector syringe (Terumo Europe) and incubated in Petri dishes, on filter paper, at $37{ }^{\circ} \mathrm{C}$ for $72 \mathrm{~h}$ in the dark. The control group consisted of ten larvae injected with $20 \mu \mathrm{l}$ sterile PBS. No more than one control larva died in any given trial. In instances where more than one larva died in the control group, the data from the entire experiment were discarded. Larval death was followed for $72 \mathrm{~h}$, at $24 \mathrm{~h}$ intervals, by visual inspection and by the lack of movement when stimulated. Three independent trials were conducted consisting of ten larvae per bacterial concentration for each specified strain. Results are presented as $\mathrm{LD}_{50}$ values, i.e. the inoculum concentrations that resulted in $50 \%$ killing of the larvae in $72 \mathrm{~h}$. Survival over time was also monitored and plotted against the serially diluted bacteria (from $1 \times 10^{0}$ to $1 \times 10^{6}$ c.f.u.) and representative graphs of three experiments are shown. To assess the virulence of cell-free supernatant (CFS), bacterial cultures were pelleted by centrifugation at 4000 r.p.m. in a microfuge for $10 \mathrm{~min}$. Supernatants were then passed through $0.22 \mu \mathrm{m}$ pore filters to remove all bacteria. An aliquot of $20 \mu \mathrm{l}$ was injected into ten larvae. Sterile broth was used as the control.

Invasion assay. The ability of Pandoraea strains to invade epithelial cells was investigated as outlined previously (Caraher et al., 2008). Lung epithelial cells $\left(16 \mathrm{HBE} 14 \mathrm{o}^{-}\right.$or CFBE410 ${ }^{-}$) were seeded on 24well plates $\left(4 \times 10^{5}\right.$ cells per well $)$ in medium containing $10 \%$ fetal bovine serum, without antibiotics, and cultured for $24 \mathrm{~h}$ at $37{ }^{\circ} \mathrm{C}$ in $5 \% \mathrm{CO}_{2}$. All bacterial strains were grown to an $\mathrm{OD}_{600}$ of 0.6 and resuspended at the appropriate dilution, as determined by growth curves, in tissue culture medium without antibiotics. These bacterial suspensions were then added to the epithelial cells at an m.o.i. of 50, as used previously. Intracellular invasion was determined over $2 \mathrm{~h}$ following antibiotic killing of extracellular bacteria with $1 \mathrm{mg}$ amikacin $\mathrm{ml}^{-1}$. The percentage invasion was determined as: (c.f.u. recovered from lysed cells/c.f.u. applied to the cells) $\times 100$.

Measurement of transepithelial electrical resistance (TER). Calu-3 cells were seeded onto Transwell polycarbonate inserts $(0.4 \mu \mathrm{m})$ and, after 1 day, the cells were maintained at an air-liquid interface in order to promote differentiation (Foster et al., 2000). TER was monitored for 14 days using an EVOM voltohmmeter (World Precision Instruments) to ensure polarization and the formation of tight junctions, and the cells were fed basolaterally on alternate days. Prior to infection of the epithelial cells, each strain was grown to an $\mathrm{OD}_{600}$ of 0.6 and $0.5 \mathrm{ml}$ of the appropriate dilution of bacterial suspension was added to the apical compartment and incubated at $37^{\circ} \mathrm{C}$. E. coli strain NCIB 9485 was used as a negative control. TER was measured at $0,1,2$ and 4 h post-infection (p.i.). 
Table 1. Summary of the origins of the Pandoraea strains and isolates used in this study and their relative $L_{50}$ values obtained in $G$. mellonella larvae compared with that of $B$. cenocepacia strain J2315

$\mathrm{LD}_{50}$ is defined as the c.f.u. required to kill $50 \%$ of the G. mellonella larvae in $72 \mathrm{~h}$. Data represent the mean of three independent trials with ten larvae per group. ND, Not determined.

\begin{tabular}{|lllc|}
\hline Species & Strain & \multicolumn{1}{c|}{ Source } & LD $_{\mathbf{5 0}}( \pm$ SEM $)$ at 72 h p.i. \\
\hline P. pulmonicola & RL7177 & CF lung (Ireland, patient 1) & $1 \times 10^{4}\left( \pm 8.7 \times 10^{3}\right)$ \\
& RL8228 & CF lung (Ireland, patient 1) & $171( \pm 159)$ \\
& RL0345 & CF lung (Ireland, patient 2) & $3.85 \times 10^{5}\left( \pm 71.5 \times 10^{4}\right)$ \\
& RL0308 & CF lung (Ireland, patient 3) & $5.12 \times 10^{5}\left( \pm 3.8 \times 10^{5}\right)$ \\
& LMG 18107 & CF lung (USA) & $1.54( \pm 1.0)$ \\
L. apista & LMG 18108 & CF lung (USA) & $31.3( \pm 30)$ \\
& LMG 16407 & CF lung (Denmark) & $8.8 \times 10^{6}\left( \pm 2.0 \times 10^{5}\right)$ \\
B. cenomenusa & LMG 16408 & CF lung (Denmark) & $1.27 \times 10^{6}\left( \pm 4.4 \times 10^{5}\right)$ \\
& LMG 18087 & CF lung (UK) & $5.2 \times 10^{6}\left( \pm 6.7 \times 10^{5}\right)$ \\
& J2315 & CF lung (USA) & $4100( \pm 3412)$ \\
\end{tabular}

Translocation studies. To examine the translocation of bacteria through the epithelial monolayer, Calu-3 cells were seeded at a density of $7.5 \times 10^{5}$ cells per insert on $3.0 \mu \mathrm{m}$ Transwell polycarbonate inserts, a pore size that enables passage of the bacteria to the basolateral chamber (Duff et al., 2006). The cells were cultured fed basolaterally on alternate days for a period of up to 21 days. After apical application of bacteria (m.o.i. 50), TER was monitored for up to $3 \mathrm{~h}$ and the basolateral medium was sampled periodically and serially diluted in Ringer's solution to determine the c.f.u. $\mathrm{ml}^{-1}$ that translocated through the cell monolayer (Duff et al., 2006).

Protease activity assay. For detection of general protease secretion, $100 \mu \mathrm{l} \mathrm{CFS} \mathrm{from} \mathrm{an} \mathrm{overnight} \mathrm{culture} \mathrm{of} \mathrm{each} \mathrm{of} \mathrm{the} \mathrm{Pandoraea} \mathrm{strains}$ was placed into wells on skimmed milk ( 3 and $10 \%$, w/v) agar plates containing nutrient agar. This method provides a convenient and clear determination of protease detection on visual inspection and is suitable for evaluating multiple bacterial strains simultaneously. Sterile broth was used as a negative control and E. coli strain NCIB 9485 and Burkholderia cenocepacia strain BC7 were used as positive controls. The plates were incubated at $37{ }^{\circ} \mathrm{C}$ for $24-48 \mathrm{~h}$ and any zones of inhibition were measured $(\mathrm{mm})$ using digital Vernier callipers (Lennox Laboratory Supplies).

Statistical analysis. Dunn's multiple comparison method was used to compare the invasiveness of individual strains with that of the negative control. A $P$ value of $<0.05$ was deemed to be significant in each case. Student's $t$-test was used to compare the TER values of bacteria-treated monolayers relative to the control at individual time points and apparent permeability coefficients, and also to compare the percentage survival of G. mellonella at specific time points following exposure to CFSs. In vivo virulence data for individual strains were compared using one-way analysis of variance (Minitab).

\section{RESULTS}

\section{Patients and isolates}

Patient 1 was an adult female patient who was colonized with $P$. pulmonicola at 36 years of age (isolate RL7177). She was chronically colonized, and a second isolate (RL8228) was obtained 9 months after the first isolate. Her forced expiratory volume $\left(\mathrm{FEV}_{1}\right)$ fell from $54 \%$ at the time of first isolation of $P$. pulmonicola to $41 \%$ at the time of the second isolation and continued to decline down to $21 \%$ at 44 months after the first $P$. pulmonicola isolation. Her hospital admissions increased from a mean of 0.6 per year prior to Pandoraea acquisition to 3 per year in the subsequent 3 years. She died 52 months after her first Pandoraea isolation, having spent the last 21 months as an inpatient.

Patient 2 was an adult female who was colonized with $P$. pulmonicola (RL0345) at 23 years of age. She was chronically colonized and a second Pandoraea isolate was obtained 8 months after the first isolation and identification. Her FEV 1 fell from 35 to $33 \%$ in the year prior to the first isolation of P. pulmonicola, and dropped from 33 to $26 \%$ in the 12 months after $P$. pulmonicola isolation. Although her hospital admissions did not change substantially after acquisition of $P$. pulmonicola (2.5 per year in the preceding years compared with 4 per year in the subsequent 12 months), she died 14 months after the first isolation of Pandoraea.

Patient 3 was also an adult female patient colonized with $P$. pulmonicola (RL0308) at 19 years of age. Her hospital admissions increased from a mean of 2 per year in the 3 years prior to Pandoraea isolation to 4 per year following Pandoraea isolation. She had a lung transplant 5 months after the first isolation of Pandoraea. Her FEV 1 was $64 \%$ at 7 months post-transplant and decreased to $39 \%$ at 11 months post-transplant. She subsequently died 2 years and 1 month after transplantation. Pandoraea was not identified in this patient post-transplant.

\section{P. pulmonicola strains are more virulent than other Pandoraea species in vivo}

We first examined the survival of G. mellonella larvae in response to the four Irish clinical $P$. pulmonicola isolates and to five other strains within the genus Pandoraea, and 
compared the results with values obtained for the $B$. cenocepacia strain J2315. This Bcc strain was chosen as it has been tested previously in a G. mellonella model (Seed \& Dennis, 2008). Although variability was observed across the genus, there were clear species-specific effects on $G$. mellonella survival. The four most virulent Pandoraea strains, with the lowest $\mathrm{LD}_{50}$ at $72 \mathrm{~h}$, were all $P$. pulmonicola strains (Table 1). Four out of six P. pulmonicola strains examined (RL7177, RL8228, LMG 18107 and LMG 18108) were quite virulent and showed comparable or greater virulence relative to that of $B$. cenocepacia strain J2315 (Table $1, P=0.147)$. The fact that the four most virulent strains included two patient isolates and two from a commercial source indicated that this effect was not biased towards the Irish clinical isolates or the commercially sourced isolates. Both P. apista strains examined (LMG 16407 and LMG 16408) exhibited high $\mathrm{LD}_{50}$ values, indicating that they were less virulent. The only $P$. pnomenusa strain examined also had a high $\mathrm{LD}_{50}$ value. Interestingly, the two sequential $P$. pulmonicola isolates (RL7177 and RL8228) obtained from patient 1 showed a dramatic reduction in $\mathrm{LD}_{50}$ over time between isolates, indicating that virulence had increased with time.

The kinetics of killing varied across the genus. Individual $P$. pulmonicola strains became more lethal with time following the injection (Fig. 1a-c), whilst P. apista and P. pnomenusa strains all had their optimal effect within $24 \mathrm{~h}$ with no significant enhancement after that time (for example, $P$. apista LMG 16408; Fig. 1d). All other P. pulmonicola strains also showed this time-dependent effect (data not shown).

\section{Cellular invasion of Pandoraea species is independent of CF phenotype}

We have shown previously that only a subset of Pandoraea strains were capable of invading lung epithelial cells. Indeed, the Irish $P$. pulmonicola strains were unusual in that the majority of these were capable of invading A549 cells (Caraher et al., 2008). To investigate the invasive potential of the $P$. pulmonicola isolates further, we examined the potential of a selection of these strains to invade lung epithelial cells with and without a CF phenotype in order to examine whether cells with a CF phenotype (CFBE41o ${ }^{-}$ cells) were more susceptible to invasion by members of this genus relative to lung epithelial cells with functional CFTR (16HBE140 ${ }^{-}$cells). An m.o.i. of 50 was used as previous studies have shown that invasion is optimal at this m.o.i. Three clinical $P$. pulmonicola isolates that were found previously to invade A549 cells (Caraher et al., 2008) were selected, including the two sequential isolates (RL7177 and RL8228) from one patient, to examine their potential to invade these two distinct cell lines $\left(16 \mathrm{HBE} 14 \mathrm{o}^{-}\right.$and CFBE410 ${ }^{-}$). These were compared with another strain shown previously to be moderately invasive, $P$. pnomenusa strain LMG 18087. Three Pandoraea strains (P. pulmonicola RL0345 and RL8228, and $P$. pnomenusa LMG 18087) were invasive in the $16 \mathrm{HBE} 14 \mathrm{o}^{-}$cells compared with the negative-control E. coli strain NCIB $9485(P<0.05$ by Dunn's multiple comparison method in each case; Fig. 2). The remaining isolate, $P$. pulmonicola RL7177, was classified as non-invasive because the invasion values were not significantly different from that of the control E. coli strain. Only two of the Pandoraea isolates, P. pulmonicola RL0345 and RL8228, invaded CFBE41o ${ }^{-}$cells (Fig. 2) when compared with the negative-control E. coli $(P<0.05)$. The other two isolates, $P$. pnomenusa LMG 18087 and $P$. pulmonicola RL7177, were not significantly more invasive in CFBE cells than E. coli strain NCIB 9485. There was no statistically significant difference in invasion of any strain into $\mathrm{CFBE}_{11 \mathrm{o}^{-}}$cells versus $16 \mathrm{HBE} 14 \mathrm{o}^{-}$cells. Although these cell lines are distinct, this study suggested that cells with a CF phenotype are no more susceptible to invasion by Pandoraea strains than those with functional CFTR. Interestingly, a consistent increase in invasive potential in the two sequential isolates, RL7177 and RL8228, in both lung epithelial cell lines suggested that these strains had become more invasive with time.

\section{P. pulmonicola strains can translocate through polarized lung epithelia}

Pandoraea has been isolated from the blood of patients (Atkinson et al., 2006; Daneshvar et al., 2001; Stryjewski et al., 2003), but the majority of strains examined previously were not invasive (Caraher et al., 2008). Therefore, we wanted to examine whether Pandoraea strains were able to invade through the lung epithelium from the apical to the basolateral side. Calu-3 cells were grown as polarized monolayers on filters with a pore size sufficiently large $(3 \mu \mathrm{m})$ to enable bacteria to pass through the filter to the basolateral chamber. Although development of a tight epithelium was slower than that observed for Calu-3 cells when cultured on the smaller pore-size $(0.4 \mu \mathrm{m})$ filters (Duff et al., 2006), the TER peaked at $150 \Omega \mathrm{cm}^{-2}$ over that of blank filters on day 21. All experiments were carried out on monolayers that had a TER of no less than $150 \Omega \mathrm{cm}^{-2}$ above that of the control well. Bacterial counts from the basolateral chamber indicated that all four strains examined appeared to translocate within $3 \mathrm{~h}$ (Fig. 3a). The P. apista strain LMG 16408 (shown previously to be non-invasive; Caraher et al., 2008) was the slowest to translocate, reaching levels in the basolateral chamber of $1 \%$ of bacteria $\left(2 \times 10^{5}\right)$ applied over the $3 \mathrm{~h}$. During this time, all the other strains appeared to translocate in excess of $1 \times 10^{6}$ c.f.u. $\mathrm{ml}^{-1}$. The strain that translocated most readily was $P$. pnomenusa strain LMG 18087.

To evaluate whether the observed translocation of Pandoraea strains was mediated by disruption of tight junctions, Calu-3 cells were grown on $0.4 \mu \mathrm{m}$ Transwell filters for 14 days, by which time the TER was routinely greater than $1000 \Omega \mathrm{cm}^{-2}$. The sequential $P$. pulmonicola isolates RL7177 and RL8228 both resulted in a significant drop in TER over time when applied to Calu-3 monolayers relative to E. coli strain NCIB $9485(P<0.015$ for both 
(a)

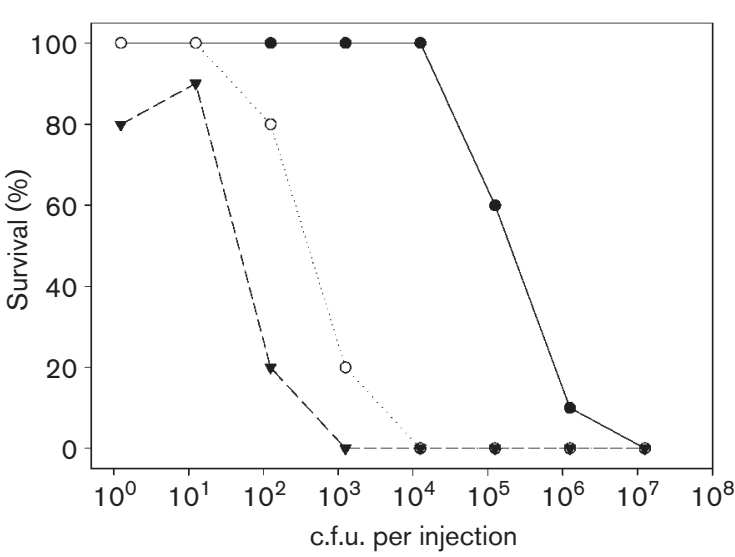

(c)

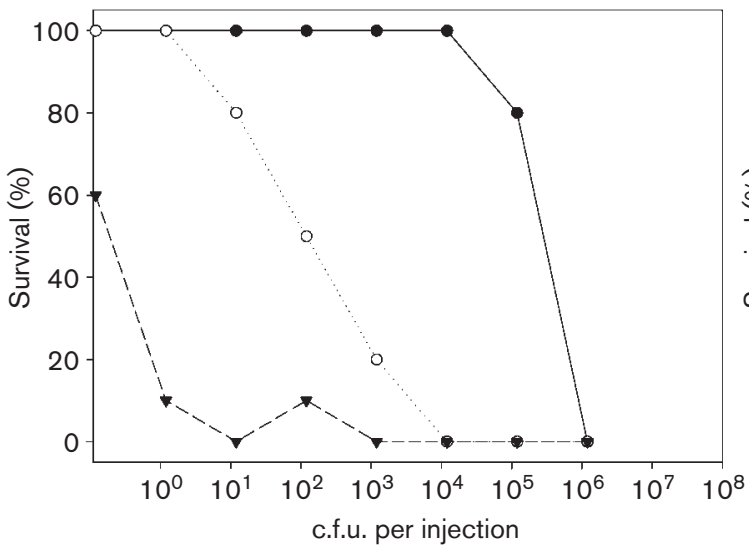

(b)

RL0345

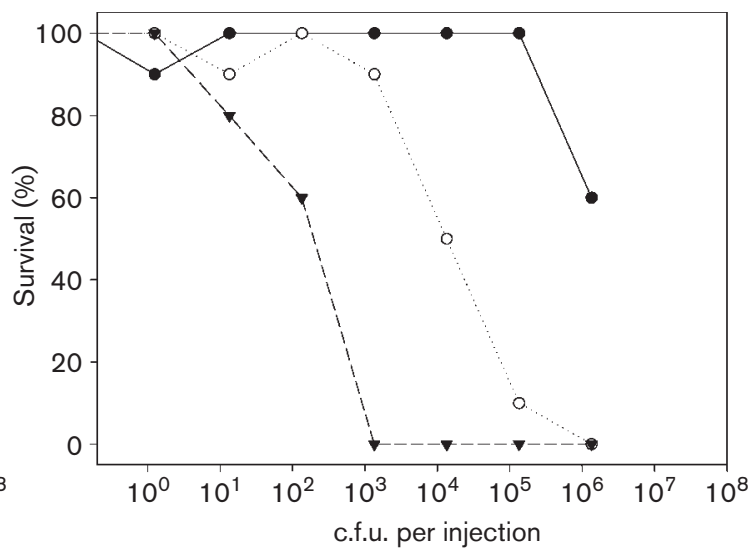

(d)

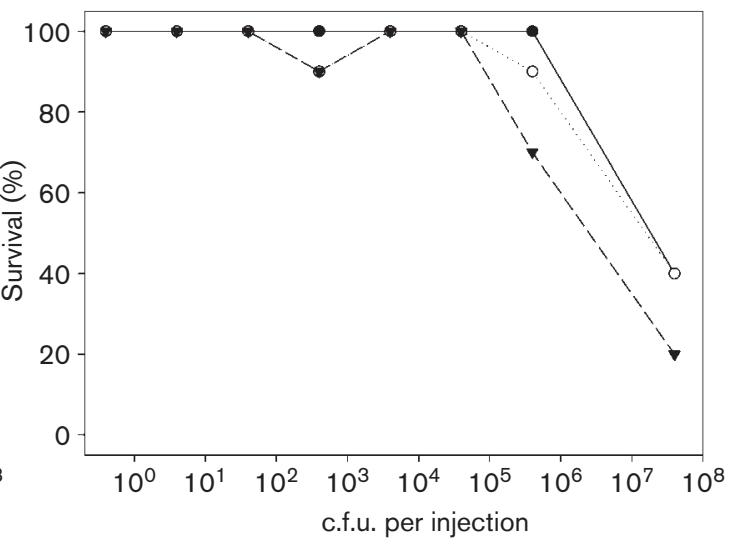

Fig. 1. Kinetics of larval killing by Pandoraea strains. Survival of G. mellonella at $24 \mathrm{~h}(\mathbf{0}), 48 \mathrm{~h}(\bigcirc)$ or $72 \mathrm{~h}(\boldsymbol{\nabla})$ following infection with P. pulmonicola strains RL8228 (a), RL0345 (b) or LMG 18108 (c), or P. apista strain LMG 16408 (d). Each dataset is from a single trial $(n=10)$, which was representative of three independent trials with the specified strain. The data are presented as percentage larval survival from ten larvae injected per trial. No more than one control larva died in any given trial.

strains; Fig. 3b). The two other strains that were shown to translocate were $P$. pnomenusa LMG 18087 and P. apista LMG 16408, but they did not cause a statistically significant reduction in TER. We have shown previously that the virulent $\mathrm{CF}$ pathogen B. cenocepacia strain BC7 disrupts tight junction integrity in polarized Calu-3 cells (Duff et al., 2006); therefore, this was included as a positive control. The drop in TER following infection with the $B$. cenocepacia strain BC7 was found to be comparable to that of the P. pulmonicola isolates RL7177 and RL8228. The TER at $4 \mathrm{~h}$ fell to $64 \pm 14 \%$ following infection with BC7 (excluded from Fig. 3b for clarity), compared with $59 \%$ for RL7177 and $66 \%$ for RL8228.

\section{Pandoraea isolates secrete proteases but these are not involved in larval killing}

Protease secretion by Pandoraea strains could play a role in disruption of tight junctions or general lung tissue damage; therefore, we wanted to determine the levels of protease secretion in a selection of the clinical Irish isolates and other strains. The protease activities of a range of Pandoraea isolates were examined on 3 or $10 \%$ skimmed milk agar plates together with those of E. coli NCIB 9485 and B. cenocepacia strain BC7, which were used as positive controls in these studies (Gingues et al., 2005; Swamy \& Goldberg, 1982). All the Irish $P$. pulmonicola isolates examined produced significant levels of protease activity. This level was comparable to all other Pandoraea strains examined and was also comparable to the levels of protease activity of E. coli strain NCIB 9485 and B. cenocepacia strain BC7 (Fig. 4).

The role of proteases in pathogenesis was examined by injecting CFS from a series of Pandoraea isolates into $G$. mellonella. In contrast to the killing of larvae by whole cells, when CFS was administered to the larvae, no killing was observed by Pandoraea strains examined up to 1 week postinjection. This clearly indicated that, in general, cellular components rather than secreted factors play a role in 


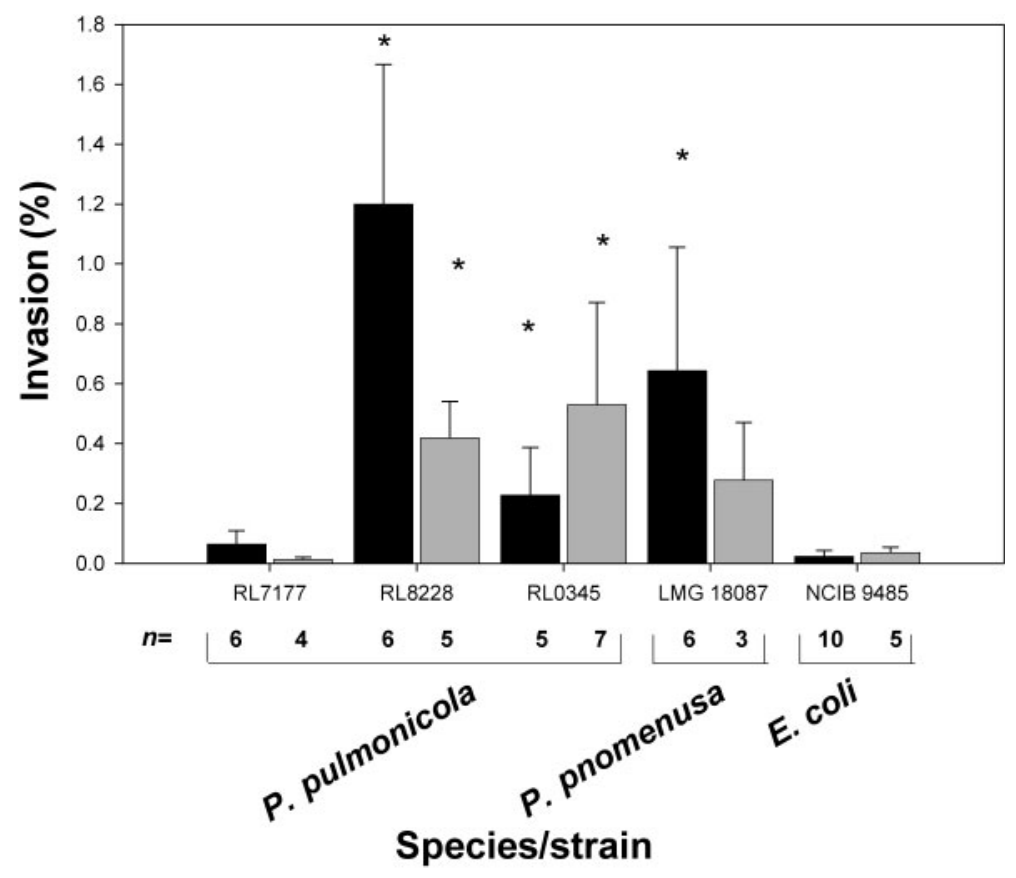

Fig. 2. Comparison of invasion by Pandoraea strains and the negative-control $E$. coli strain NCIB 9485 in two lung epithelial cell lines, $16 \mathrm{HBE}_{140} \mathrm{o}^{-}$(black bars) and CFBE410 (grey bars). Results are shown as percentage invasion (c.f.u. obtained after cell lysis as a percentage of c.f.u. applied) of at least five independent experiments for $16 \mathrm{HBE} 14 \mathrm{o}^{-}$ cells and at least three independent experiments for CFBE410 ${ }^{-}$, shown as means \pm SEM. *, $P<0.05$ (invasion significantly greater than that of $E$. coli, as determined by Dunn's multiple comparison test).

killing among members of the genus Pandoraea. This contrasted with the effects of CFS from B. cenocepacia strain J2315 (Fig. 5a), which resulted in significant killing of G. mellonella larvae within $72 \mathrm{~h} \quad(70 \%$ survival). Interestingly, in spite of the lack of effect on survival, the CFS of certain strains, such as $P$. pulmonicola strains LMG 18107 and LMG 18108 and P. pnomenusa strain LMG 18087, had a reversible pathogenic effect on the larvae. The larvae underwent melanization within $30 \mathrm{~min}$ (Fig. 5c) and subsequently recovered without any effect on survival. This melanization was never observed in the control larvae injected with LB broth only (Fig. 5b). However, melanization always preceded death of the larvae that were injected with whole cells (Fig. 5d).

\section{DISCUSSION}

Pandoraea species are a relatively recently identified pathogen among CF and non-CF patients (LiPuma, 2003; Pimentel \& MacLeod, 2008; Stryjewski et al., 2003). Although Pandoraea has been isolated from the blood and sputum samples of patients, very little is known about its virulence or mechanisms of pathogenicity. P. pulmonicola is the most commonly identified Pandoraea species among Irish CF patients. However, there is very little known about this species, in particular in terms of the outcomes of infected patients or its pathogenicity. Therefore we have focused, although not exclusively, on this species. All three patients from whom $P$. pulmonicola was isolated were female. This was an unavoidable bias in this study, as there were not enough patients to allow a more gender-balanced approach. In our experience, no gender bias has been observed in Pandoraea colonization.
First, in order to compare the potential virulence of Pandoraea species and strains, we evaluated nine strains in an in vivo virulence model. The larvae of G. mellonella have been used recently to evaluate a series of Bcc strains for their virulence and their responses to phage therapies (Seed \& Dennis, 2008, 2009). They have also been used to study the virulence of other bacterial and fungal pathogens (Jander et al., 2000; Reeves et al., 2004). The host response of G. mellonella to infection shares many similarities with that of vertebrates (Kavanagh \& Reeves, 2004), making it a cheaper alternative to other in vivo models. It has advantages over other in vivo models, such as Caenorhabditis elegans, as Galleria larvae can survive at $37^{\circ} \mathrm{C}$. The pattern of survival of G. mellonella following infection with Pandoraea strains was species specific. Although the in vivo studies were limited in that they were performed in a single model, it was clear that the $P$. pulmonicola strains were among the most virulent of the three species tested in this model, with the other species, P. apista and P. pnomenusa, being only weakly virulent (Table 1).

P. pulmonicola was also the species in which the majority of strains displayed a potential for epithelial cell uptake (Caraher et al., 2008). Therefore, in order to investigate invasion further as a mechanism of virulence within these Pandoraea strains, we compared invasion in cells with and without a CF phenotype. Although the magnitude of invasion into CFBE cells was less in general, the pattern of invasion (i.e. invasive or not) of Pandoraea isolates into both independent cell lines was comparable with invasion of A549 cells. In particular, two of the Irish P. pulmonicola isolates, RL0345 and RL8228, which showed considerable invasive capacity of A549 cells, were also found to be significantly invasive in both $16 \mathrm{HBE} 14 \mathrm{o}^{-}$and CFBE41o ${ }^{-}$ epithelial cell models. This demonstrates the ability of certain 

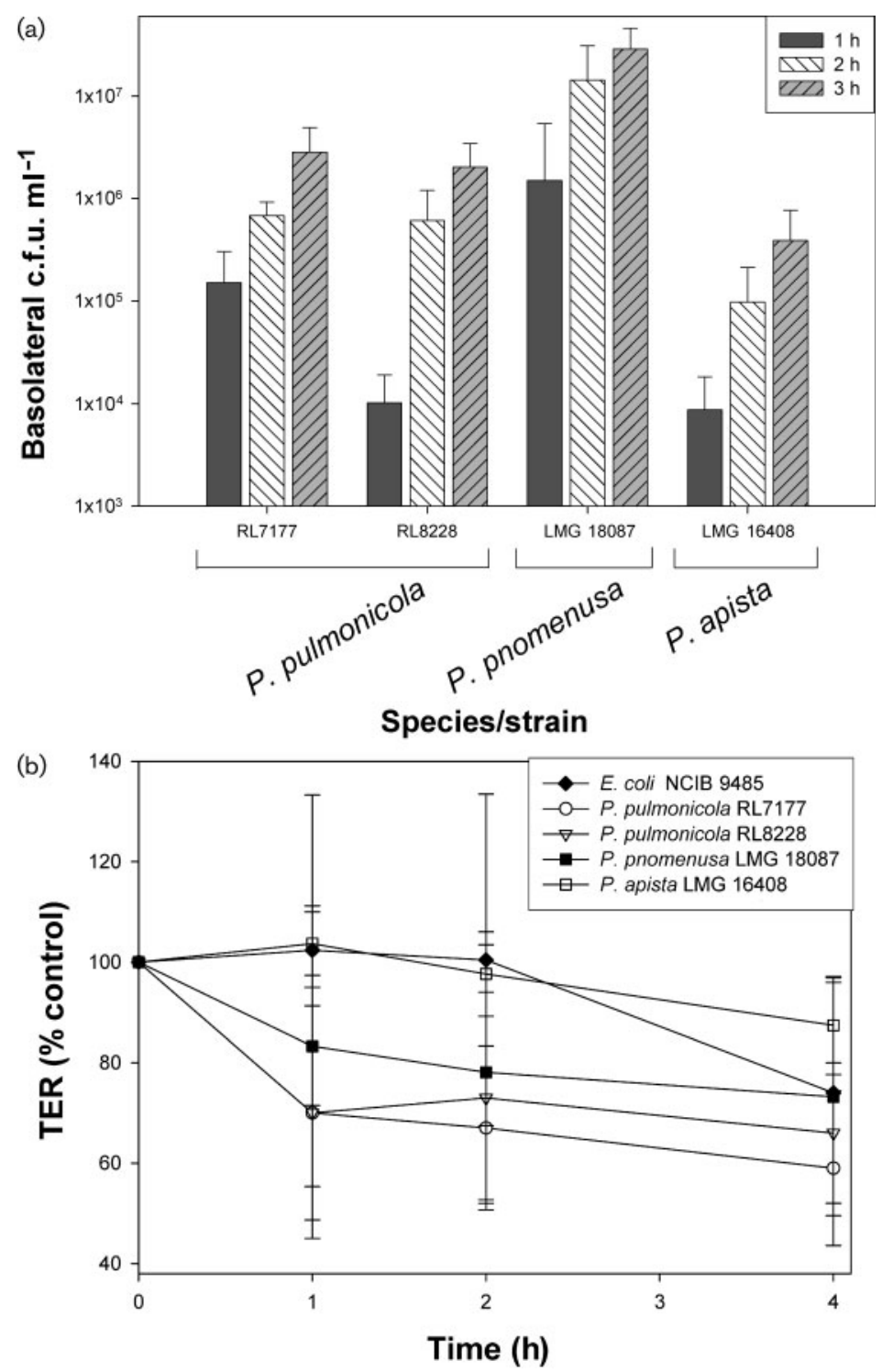

Fig. 3. Translocation of Pandoraea strains through polarized Calu-3 epithelial monolayers. (a) Cumulative bacterial counts taken from the basolateral chamber of Calu-3 cell monolayers grown on $3 \mu \mathrm{m}$ Transwell filters at 1,2 and $3 \mathrm{~h}$ after infection with Pandoraea strains expressed as total c.f.u. translocated $\mathrm{ml}^{-1}$. The results are the means \pm SEM from at least two independent experiments. (b) Effect of Pandoraea strains on TER at 0, 1, 2 and $4 \mathrm{~h}$ p.i., presented as the percentage of the $E$. coli control $(1092 \pm 225)$ and representing the means \pm SEM of at least three independent experiments.
Pandoraea isolates to invade three independent lung epithelial cells and indicates that invasion is strain-specific rather than cell-phenotype dependent. The lack of enhanced susceptibility to invasion of cells with a CF phenotype over non-CF cells agrees with a recent summary of nine Pandoraea bacteraemia cases reported (Pimentel \& MacLeod, 2008), at least three of which were identified as being in non-CF patients. The finding that $P$. pulmonicola isolate RL8228 was both more invasive in human lung cells and more virulent to larvae than the earlier isolate (RL7177) from the same patient suggests that invasion plays a role in pathogenesis for some members of the species. However, the finding that the two other P. pulmonicola strains (LMG 18107 and LMG 18108) were very virulent towards the larvae but were not shown previously to be invasive indicates that other mechanisms of pathogenicity are also at play.

In order to explore mechanisms of lung invasion further, the ability of a selection of isolates to disrupt epithelial integrity prior to entering the bloodstream was examined. Human lung epithelial cells such as Calu-3 show permeability properties that are comparable to those of native lung epithelia and therefore are a useful model for studying the absorption barrier of the lung (Forbes \& Ehrhardt, 2005). We and others have shown that Bcc strains can disrupt the tight junction integrity of, and penetrate through, lung epithelial monolayers (Duff et al., 2006; Kim et al., 2005). Although all four Pandoraea isolates tested had the ability to translocate through the monolayer, albeit at different rates, two of the strains examined translocated without having any effect on TER (Fig. 3). This suggests that these pathogens move through the monolayer via an alternative route to the paracellular pathway.

Despite the diversity across the genus, all Pandoraea strains tested secreted significant levels of protease and the three Pandoraea species examined showed comparable protease 


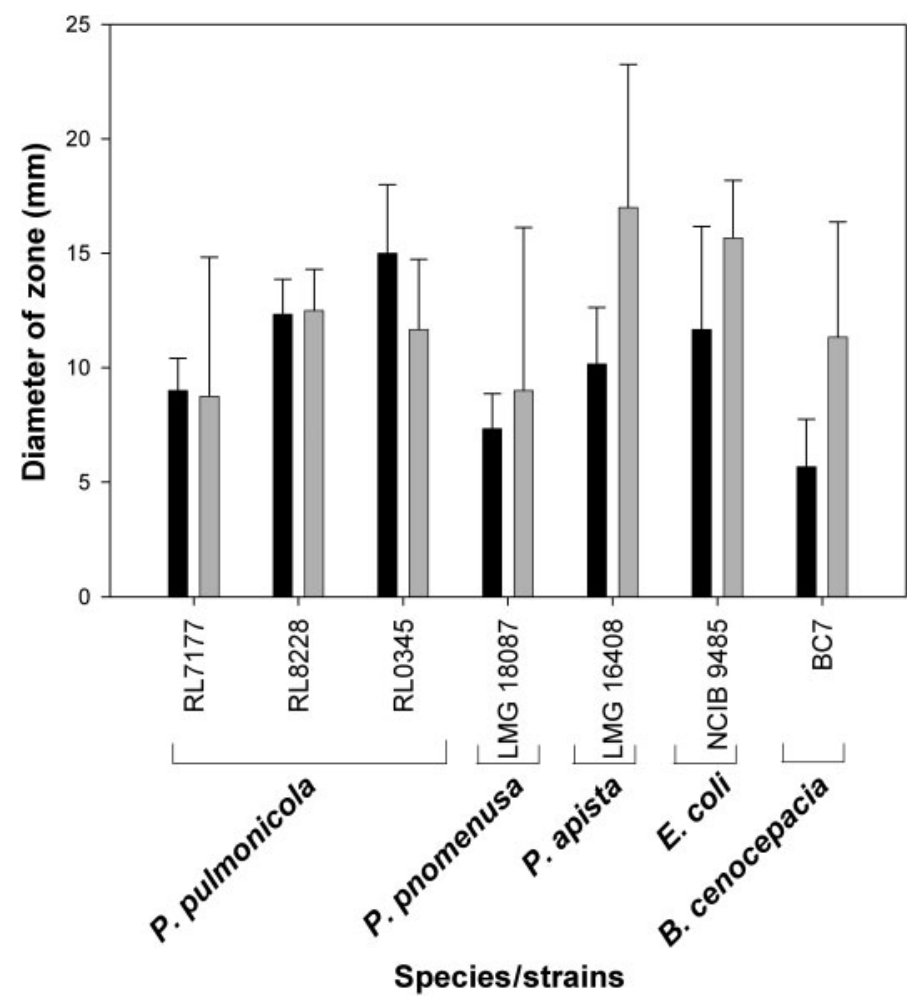

Fig. 4. Protease secretion from Pandoraea species as determined on skimmed milk agar plates. Bars represent zones of clearance ( \pm SD) measured following $48 \mathrm{~h}$ incubation of Pandoraea cell CFS on $3 \%$ (black bars) or $10 \%$ (grey bars) skimmed milk plates from three independent experiments.

activities. Bacterial proteases have been implicated in a number of pathogenic processes, including disruption of epithelial integrity, inflammation and tissue damage. However, the lack of effect of CFS in the in vivo model suggested that secreted proteases, in general, may not play a major role in pathogenesis. Furthermore, the lack of correlation between protease activity and tight junction disruption suggested that proteases are not likely to be responsible for this effect. In contrast, members of the closely related $\mathrm{Bcc}$ and also Peudomonas aeruginosa express metalloproteases, which have been shown to be involved in virulence (McKevitt \& Woods, 1984; Kooi et al., 2006). In addition, serine proteases have been identified in $B$. cenocepacia, but, although one of these has been shown to play a role in iron acquisition, their roles in pathogenicity have not been confirmed (Flannagan et al., 2007; Whitby et al., 2006). The relatively high level of general protease secretion across the genus is interesting, and the identity of these proteases is currently being investigated. It remains a possibility that specific proteases may play a role in the virulence of Pandoraea species. Overall, the lack of effect of CFS on G. mellonella survival for any species within the genus indicates that the whole cells are the main mediator of virulence rather than secreted exoproducts, strengthening the suggestion for direct interaction between the bacterial cells and the host cells as part of the virulence process.

This study showed that the genus Pandoraea is quite divergent in terms of virulence characteristics; indeed, it was named for the potential Pandora's box of genetic diversity associated with these organisms (Vandamme et al., 2000). In general, P. pulmonicola strains are the most invasive, and the majority of strains from this species also showed the most virulence in the in vivo model. However, to the best of our knowledge, there have been no reported cases of $P$. pulmonicola-related bacteraemia. Given the small numbers involved, it was difficult to correlate in vitro behaviour with the clinical outcome in patients; however, it is significant that all $P$. pulmonicolacolonized patients from which the isolates in this study were obtained have since died. Further studies will be required with a greater number of isolates to draw any definite conclusions.

The $P$. apista strains examined showed the lowest virulence in G. mellonella, were non-invasive and P. apista strain LMG 16408 was the slowest to translocate across the polarized lung epithelium. However, this species has been associated with at least two cases of septicaemia (Daneshvar et al., 2001; Johnson et al., 2004). Only one P. pnomenusa strain, LMG 18087 , was included in this study and, although invasive in lung cells expressing CFTR, it was not invasive in cells with a CF phenotype. This strain showed the greatest level of transepithelial translocation among the four isolates examined. Interestingly, four cases of $P$. pnomenusa-associated bacteraemia have been reported (Daneshvar et al., 2001; Stryjewski et al., 2003). Indeed, all three isolates identified as P. pnomenusa by Daneshvar et al. (2001) were isolated from blood, which led the authors to suggest that this species might have increased potential for invasive disease. The mechanism of $P$. pnomenusa translocation is not linked to 
(a)

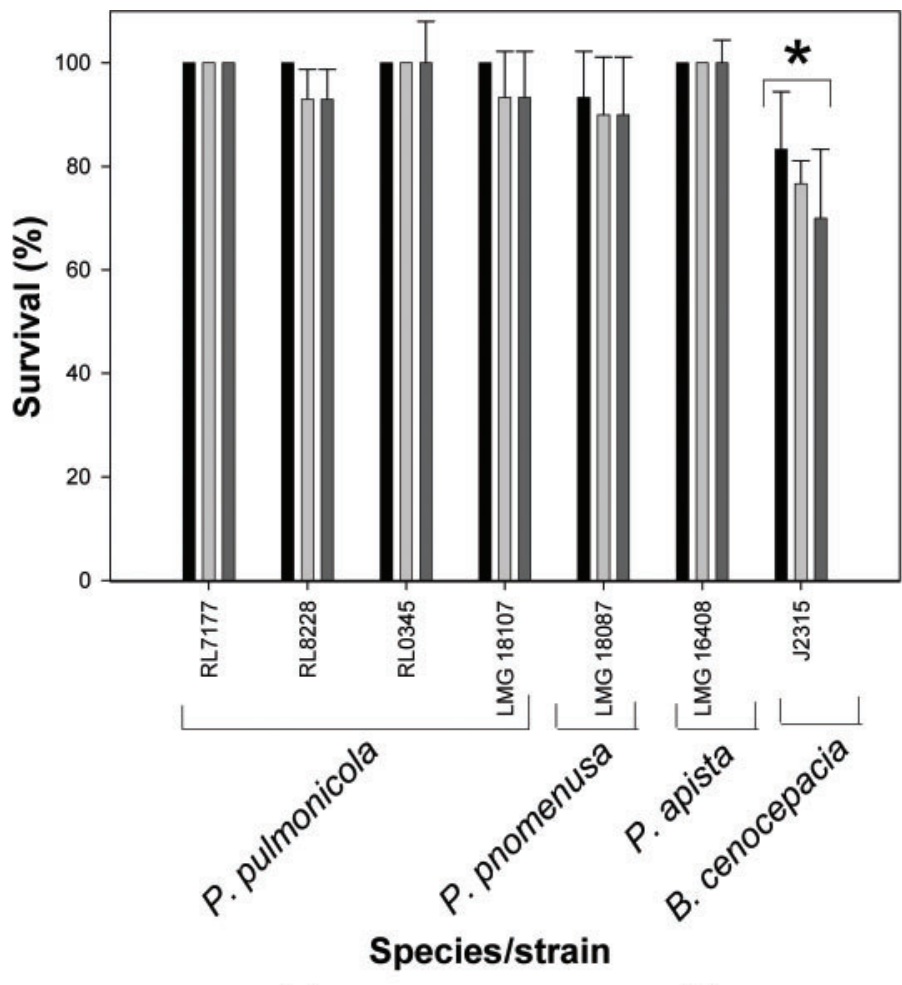

(b)

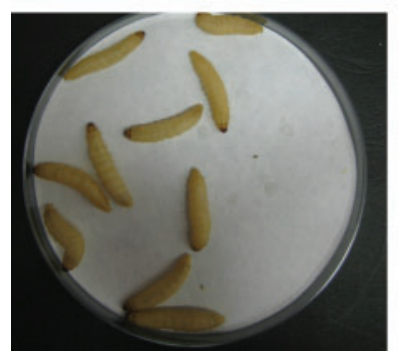

(c)

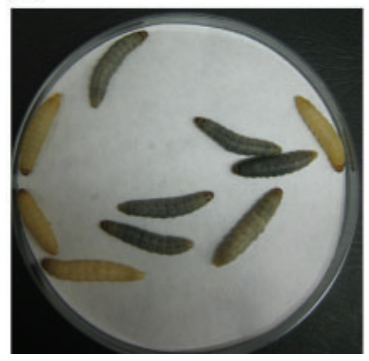

(d)

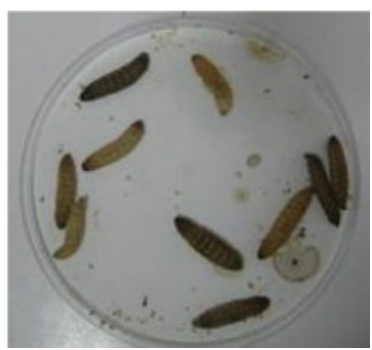

Fig. 5. Effect of CFS on survival of G. mellonella. (a) Bars represent the percentage survival of ten larvae at 24 (black bars), 48 (light-grey bars) and 72 (dark-grey bars) h. Each bar represents the mean \pm SEM of three independent experiments. ${ }^{*}, P<0.05$, relative to LB-injected control group. (b-d) Effect of $P$. pulmonicola RL7177 CFS on G. mellonella larvae 30 min after injection with tryptic soy broth only (control; b), 30 min after injection with CFS from LMG 18108 (c) and 48 h after injection with whole cells, showing killed larvae among the melanized larvae $(d)$.

tight junction opening; therefore, further analysis of this species is warranted.

The changes in the sequential P. pulmonicola isolates RL7177 and RL8228 are noteworthy. The later isolate was more invasive in lung epithelial cells and more virulent in larvae. Furthermore, in our previous study, we demonstrated that the early isolate, RL7177, was the only Pandoraea strain out of 19 examined that was capable of biofilm formation (Caraher et al., 2008), suggesting that this strain also underwent a mucoid to non-mucoid change over time. This change from a mucoid to a non-mucoid state has also been observed in Bcc isolates from chronically infected patients (Zlosnik et al., 2008), indicating that the same phenotype change arises among $P$. pulmonicola isolates; however, more sequential isolates would need to be studied to confirm this. In addition, further studies of the existing sequential isolates are being performed to elucidate the apparent alteration in virulence over time.

Overall, it was clear that P. pulmonicola strains showed comparable in vivo virulence to B. cenocepacia strain J2315 in this G. mellonella model. A subset of P. pulmonicola strains were shown to invade lung epithelial cells irrespective of CF status, and the four strains examined showed the potential to translocate across the lung epithelium. In addition, although protease secretion was a common feature among all strains examined, there is no clear evidence to date that this may be a virulence mechanism in this genus. It is likely that individual species within the 
genus Pandoraea have the potential to express very different mechanisms of pathogenesis, as demonstrated by differences in intracellular invasion, tight junction disruption and larval killing. This is comparable to the situation in Bcc strains, which appear to use different virulence mechanisms to compete for survival in different environments and hosts (Uehlinger et al., 2009). There are limited clinical data published on Pandoraea infections, and the numbers of isolates evaluated in this study were also limited; therefore, it is difficult to draw clear conclusions regarding the mechanisms of pathogenicity and the effects on patients. In addition, major host-related factors are also at play and, given that many Pandoraea-colonized patients are also co-colonized with other pathogens, the interplay between competing species must also be considered. However, it is clear that $P$. pulmonicola does contribute to the deterioration of colonized patients and has a direct interaction with lung epithelial cells.

\section{ACKNOWLEDGEMENTS}

This study was supported by PRTLI Cycle 4 (supported by the European Union Regional Development Plan, the Irish Government National Development Plan 2007-2013 and administered by the Higher Education Authority in Ireland) and by the Technology Sector Research Post-graduate R\&D Skills Programme (PRDSP), Strand I. The authors would also like to thank Dr Dieter Gruenert for kindly providing us with the $16 \mathrm{HBE}^{-} \mathrm{o}^{-}$and $\mathrm{CFBE} 41 \mathrm{o}^{-}$cells. We are grateful to James Reilly, ITT, Dublin, Ireland, for advice on statistical analysis of the data. In addition, we would like to thank Professor Philip Murphy and Jonathan Collins, National CF Reference Laboratory at Adelaide Meath Incorporating the National Children's Hospital, Tallaght, Ireland, for providing the Irish P. pulmonicola isolates.

\section{REFERENCES}

Atkinson, R. M., Lipuma, J. J., Rosenbluth, D. B. \& Dunne, W. M., Jr (2006). Chronic colonization with Pandoraea apista in cystic fibrosis patients determined by repetitive-element-sequence PCR. J Clin Microbiol 44, 833-836.

Caraher, E., Collins, J., Herbert, G., Murphy, P. G., Gallagher, C. G., Crowe, M. J., Callaghan, M. \& McClean, S. (2008). Evaluation of in vitro virulence characteristics of the genus Pandoraea in lung epithelial cells. J Med Microbiol 57, 15-20.

Daneshvar, M. I., Hollis, D. G., Steigerwalt, A. G., Whitney, A. M., Spangler, L., Douglas, M. P., Jordan, J. G., MacGregor, J. P., Hill, B. C. \& other authors (2001). Assignment of CDC weak oxidizer group 2 (WO-2) to the genus Pandoraea and characterization of three new Pandoraea genomospecies. J Clin Microbiol 39, 1819-1826.

Duff, C., Murphy, P. G., Callaghan, M. \& McClean, S. (2006). Differences in invasion and translocation of Burkholderia cepacia complex species in polarised lung epithelial cells in vitro. Microb Pathog 41, 183-192.

Flannagan, R. S., Aubert, D., Kooi, C., Sokol, P. A. \& Valvano, M. A. (2007). Burkholderia cenocepacia requires a periplasmic HtrA protease for growth under thermal and osmotic stress and for survival in vivo. Infect Immun 75, 1679-1689.

Forbes, B. \& Ehrhardt, C. (2005). Human respiratory epithelial cell culture for drug delivery applications. Eur J Pharm Biopharm 60, 193205.
Foster, K. A., Avery, M. L., Yazdanian, M. \& Audus, K. L. (2000). Characterization of the Calu- 3 cell line as a tool to screen pulmonary drug delivery. Int J Pharm 208, 1-11.

Gingues, S., Kooi, C., Visser, M. B., Subsin, B. \& Sokol, P. A. (2005). Distribution and expression of the ZmpA metalloprotease in the Burkholderia cepacia complex. J Bacteriol 187, 8247-8255.

Jander, G., Rahme, L. G. \& Ausubel, F. M. (2000). Positive correlation between virulence of Pseudomonas aeruginosa mutants in mice and insects. J Bacteriol 182, 3843-3845.

Johnson, L. N., Han, J. Y., Moskowitz, S. M., Burns, J. L., Qin, X. \& Englund, J. A. (2004). Pandoraea bacteremia in a cystic fibrosis patient with associated systemic illness. Pediatr Infect Dis J 23, 881882.

Jørgensen, I. M., Johansen, H. K., Frederiksen, B., Pressler, T., Hansen, A., Vandamme, P., Høiby, N. \& Koch, C. (2003). Epidemic spread of Pandoraea apista, a new pathogen causing severe lung disease in cystic fibrosis patients. Pediatr Pulmonol 36, 439-446.

Kavanagh, K. \& Reeves, E. P. (2004). Exploiting the potential of insects for in vivo pathogenicity testing of microbial pathogens. FEMS Microbiol Rev 28, 101-112.

Kim, J. Y., Sajjan, U. S., Krasan, G. P. \& LiPuma, J. J. (2005). Disruption of tight junctions during traversal of the respiratory epithelium by Burkholderia cenocepacia. Infect Immun 73, 7107-7112.

Kooi, C., Subsin, B., Chen, R., Pohorelic, B. \& Sokol, P. A. (2006). Burkholderia cenocepacia $\mathrm{ZmpB}$ is a broad-specificity zinc metalloprotease involved in virulence. Infect Immun 74, 4083-4093.

LiPuma, J. J. (2003). Burkholderia and emerging pathogens in cystic fibrosis. Semin Respir Crit Care Med 24, 681-692.

McKevitt, A. I. \& Woods, D. E. (1984). Characterization of Pseudomonas cepacia isolates from patients with cystic fibrosis. J Clin Microbiol 19, 291-293.

Pimentel, J. D. \& MacLeod, C. (2008). Misidentification of Pandoraea sputorum isolated from sputum of a patient with cystic fibrosis and review of Pandoraea species infections in transplant patients. J Clin Microbiol 46, 3165-3168.

Reeves, E. P., Messina, C. G., Doyle, S. \& Kavanagh, K. (2004). Correlation between gliotoxin production and virulence of Aspergillus fumigatus in Galleria mellonella. Mycopathologia 158, 73-79.

Schneider, I., Queenan, A. M. \& Bauernfeind, A. (2006). Novel carbapenem-hydrolyzing oxacillinase OXA-62 from Pandoraea pnomenusa. Antimicrob Agents Chemother 50, 1330-1335.

Seed, K. D. \& Dennis, J. J. (2008). Development of Galleria mellonella as an alternative infection model for the Burkholderia cepacia complex. Infect Immun 76, 1267-1275.

Seed, K. D. \& Dennis, J. J. (2009). Experimental bacteriophage therapy increases survival of Galleria mellonella larvae infected with clinically relevant strains of the Burkholderia cepacia complex. Antimicrob Agents Chemother 53, 2205-2208.

Shen, B. Q., Finkbeiner, W. E., Wine, J. J., Mrsny, R. J. \& Widdicombe, J. H. (1994). Calu-3: a human airway epithelial cell line that shows cAMP-dependent $\mathrm{Cl}^{-}$secretion. Am J Physiol 266, L493-L501.

Stryjewski, M. E., LiPuma, J. J., Messier, R. H., Jr, Reller, L. B. \& Alexander, B. D. (2003). Sepsis, multiple organ failure, and death due to Pandoraea pnomenusa infection after lung transplantation. J Clin Microbiol 41, 2255-2257.

Swamy, K. H. \& Goldberg, A. L. (1982). Subcellular distribution of various proteases in Escherichia coli. J Bacteriol 149, 1027-1033.

Uehlinger, S., Schwager, S., Bernier, S. P., Riedel, K., Nguyen, D. T., Sokol, P. A. \& Eberl, L. (2009). Identification of specific and universal virulence factors in Burkholderia cenocepacia strains by using multiple infection hosts. Infect Immun 77, 4102-4110. 
Vandamme, P., Mahenthiralingam, E., Holmes, B., Coenye, T., Hoste, B., De Vos, P., Henry, D. \& Speert, D. P. (2000). Identification and population structure of Burkholderia stabilis sp. nov. (formerly Burkholderia cepacia genomovar IV). J Clin Microbiol 38, 1042-1047.

Vonberg, R. P. \& Gastmeier, P. (2005). Isolation of infectious cystic fibrosis patients: results of a systematic review. Infect Control Hosp Epidemiol 26, 401-409.
Whitby, P. W., Vanwagoner, T. M., Springer, J. M., Morton, D. J., Seale, T. W. \& Stull, T. L. (2006). Burkholderia cenocepacia utilizes ferritin as an iron source. J Med Microbiol 55, 661-668.

Zlosnik, J. E., Hird, T. J., Fraenkel, M. C., Moreira, L. M., Henry, D. A. \& Speert, D. P. (2008). Differential mucoid exopolysaccharide production by members of the Burkholderia cepacia complex. J Clin Microbiol 46, 1470-1473. 